\title{
ON $L_{1}$-BIHARMONIC TIMELIKE HYPERSURFACES IN PSEUDO-EUCLIDEAN SPACE $\mathbb{E}_{1}^{4}$
}

\author{
FIROOZ PASHAIE
}

\begin{abstract}
A well-known conjecture of Bang-Yen Chen says that the only biharmonic submanifolds in the Euclidean spaces are minimal ones. In this paper, we consider an extended condition (namely, $L_{1}$-biharmonicity) on non-degenerate timelike hypersurfaces of the pseudo-Euclidean space $\mathbb{E}_{1}^{4}$. A Lorentzian hypersurface $x: M_{1}^{3} \rightarrow \mathbb{E}_{1}^{4}$ is called $L_{1}$-biharmonic if it satisfies the condition $L_{1}^{2} x=0$, where $L_{1}$ is the linearized operator associated with the first variation of 2 th mean curvature vector field on $M_{1}^{3}$. According to the multiplicities of principal curvatures, the $L_{1}$-extension of Chen's conjecture is proved for Lorentzian hypersurfaces with constant ordinary mean curvature in the pseudo-Euclidean space $\mathbb{E}_{1}^{4}$. Additionally, we show that there is no proper $L_{1}$-biharmonic $L_{1}$-finite type connected orientable Lorentzian hypersurface in $\mathbb{E}_{1}^{4}$.
\end{abstract}

\section{Introduction}

Biharmonic surfaces in the Euclidean spaces play fundamental roles in the theory of elastics and also in fluid mechanics. For instance, the solutions of plane elastic problems can be stated in terms of biharmonic functions. In general, biharmonic maps appear in the theory of partial differential equations as the solutions of some 4-order strongly elliptic semilinear equations. Also, one can find the role of biharmonic Bezier surfaces in computational geometry. It is clear that the harmonic maps are biharmonic but not vis versa. By definition, a biharmonic map, which is not harmonic, is said to be proper-biharmonic. For instance, in the homotopy class of Brower of degree \pm 1 , one may not find a harmonic map as $\mathbb{T}^{2} \rightarrow \mathbb{S}^{2}$. Although, there exists a proper-biharmonic map from $\mathbb{T}^{2}$ into $\mathbb{S}^{2}$ (introduced in [10]). From a geometric point of view, the variational problem associated with the bi-energy functional on the set of Riemannian metrics on a domain has given rise to the biharmonic stress-energy tensor. It is useful to obtain new examples of proper-biharmonic maps for the study of submanifolds with certain geometric properties, like pseudo-umbilical and parallel submanifolds.

A differential geometric motivation of the subject of biharmonic maps is a well-known result of Bang-Yen Chen (1987) which states that there is no proper-biharmonic surface in the 2010 Mathematics Subject Classification. Primary: 53-02, 53C40, 53C42; Secondary 58G25. Key words and phrases. Lorentz hypersurface, Biharmonic, $L_{1}$-biharmonic, 1-minimal. 
Euclidean 3-spaces $\mathbb{E}^{3}$. Later on, Dimitrić in his doctoral thesis proved that any biharmonic hypersurface in $\mathbb{E}^{m}$ with at most two distinct principal curvatures is minimal ([9]). In 1995, Hasanis and Vlachos proved an extension of Chen's result to the hypersurfaces in Euclidean 4-spaces ([11]). In 2013, Akutagawa and Maeta ([1]) have generalized Chen's conjecture on biharmonic submanifolds Euclidean spaces. On the other hand, Chen himself had found a nice relationship between the finite type hypersurfaces and biharmonic ones. The theory of finite type hypersurfaces is a well-known subject interested by Chen (for instance, in [5, 6]) and also followed by L.J. Alias, S.M.B. Kashani and others. In [12], Kashani has studied the notion of $L_{1}$-finite type Euclidean hypersurfaces as an extension of finite type ones. One can see main results in Chapter 11 of Chen's book ([5]).

The map $L_{k}$, as an extension of the Laplace operator $L_{0}=\Delta$, stands for the linearized operator of the first variation of the $(k+1)$ th mean curvature of the hypersurface (see, for instance, $[2,13,17,18,20])$. This operator is defined by $L_{k}(f)=\operatorname{tr}\left(P_{k} \circ \nabla^{2} f\right)$ for any $f \in C^{\infty}(M)$, where $P_{k}$ denotes the $k$-th Newton transformation associated to the second fundamental from of the hypersurface and $\nabla^{2} f$ is the hessian of $f$.

As an extended case, a hypersurface $x: M_{p}^{3} \rightarrow \mathbb{E}_{1}^{4}$, whose mean curvature vector field is an eigenvector of the Laplace operator $\Delta$, has been studied, for instance, in $[7,8]$ for the Euclidean case (where, $p=s=0$ ), and for the Lorentz case in [3, 4] (for $s=1$ and $p=0,1$ ). It is interesting to generalize the definition of biharmonic hypersurface by replacing $\Delta$ by $L_{1}$. Recently, in [15], we have studied the $L_{1}$-biharmonic spacelike hypersurfaces in 4-dimentional Minkowski space $\mathbb{E}_{1}^{4}$. In this paper, we study the Lorentzian hypersurfaces in Einstein space $\mathbb{E}_{1}^{4}$. We pay attention to proper- $L_{1}$-biharmonic timelike hypersurfaces as $x: M_{1}^{3} \rightarrow \mathbb{E}_{1}^{4}$ with three mutually distinct principal curvatures and constant mean curvature. Now, we state here our main results.

Theorem 1.1. Each $L_{1}$-biharmonic connected orientable timelike hypersurface in the LorentzMinkowski 4-space with three distinct real principal curvatures and constant mean curvature is 1-minimal.

Theorem 1.2. Each $L_{1}$-biharmonic connected orientable timelike hypersurface in the LorentzMinkowski 4-space with two distinct complex and one constant real principal curvatures and constant mean curvature is 1-minimal.

Theorem 1.3. Each $L_{1}$-biharmonic connected orientable timelike hypersurface in the LorentzMinkowski 4-space with at most two distinct principal curvatures is 1-minimal.

Theorem 1.4. There is no $L_{1}$-biharmonic $L_{1}$-finite type connected orientable Lorentzian hypersurface in the Lorentz-Minkowski4-space. 


\section{Preliminaries}

In this section, we recall preliminaries from [2, 13, 14] and [16]-[19]. The 4-dimensional pseudo-Euclidean space, denoted by $\mathbb{E}_{1}^{4}$, is the real vector space $\mathbb{R}^{4}$ endowed with the indefinite inner product defined by $\langle x, y\rangle:=-x_{1} y_{1}+\Sigma_{i=2}^{4} x_{i} y_{i}$, for every $x, y \in \mathbb{R}^{4}$. In $\mathbb{E}_{1}^{4}$, any nondegenerate hypersurface $M_{p}^{3}$ can be endowed with a Riemannian or a Lorentzian induced metric of index $p=0$ or $p=1$, according to whether the induced metric is positive definite or indefinite.

Throughout the paper, we study on a Lorentz hypersurface in $\mathbb{E}_{1}^{4}$, denoted by an isometric immersion $x: M_{1}^{3} \rightarrow \mathbb{E}_{1}^{4}$. The symbols $\tilde{\nabla}$ and $\bar{\nabla}$ stand for the Levi-Civita connection on $M_{1}^{3}$ and $\mathbb{E}_{1}^{4}$, respectively. For every tangent vector fields $X$ and $Y$ on $M$, the Gauss formula is given by $\bar{\nabla}_{X} Y=\tilde{\nabla}_{X} Y+<S X, Y>\mathbf{n}$, for every $X, Y \in \chi(M)$, where, $\mathbf{n}$ is a (locally) unit normal vector field on $M$ and $S$ is the shape operator of $M$ relative to $\mathbf{n}$. For each non-zero vector $X \in \mathbb{E}_{1}^{4}$, the real value $\langle X, X>$ may be a negative, zero or positive number and then, the vector $X$ is said to be time-like, light-like or space-like, respectively. According to whether the induced metric on a nondegenerate hypersurface $M_{r}^{3}$ of index $r$ in $\mathbb{E}_{1}^{4}$ is positive definite or indefinite, $M_{r}^{3}$ is called Riemannian (when $r=0$ ) or Lorentzian (when $r=1$ ), and therefore every normal vector on $M_{r}^{3}$ is time-like or light-like, respectively.

For a Lorentzian vector space $V_{1}^{3}$, a basis $\mathscr{B}:=\left\{e_{1}, e_{2}, e_{3}\right\}$ is said to be orthonormal if it satisfies $<e_{i}, e_{j}>=\epsilon_{i} \delta_{i}^{j}$ for $i, j=1,2,3$, where $\epsilon_{1}=-1$ and $\epsilon_{i}=1$ for $i=2,3$. As usual, $\delta_{i}^{j}$ stands for the Kronecker function. $\mathscr{B}$ is called pseudo-orthonormal if it satisfies $<e_{1}, e_{1}>=<$ $e_{2}, e_{2}>=0,<e_{1}, e_{2}>=-1$ and $<e_{i}, e_{j}>=\delta_{i}^{j}$, for $i=1, \ldots, n$ and $j=3, \ldots, n$.

As well-known, the shape operator of the Lorentzian hypersurface $M_{1}^{3}$, as a self-adjoint linear map on the tangent space of $M_{1}^{3}$, can be put into one of four possible canonical matrix forms, usually denoted by I, II, III and IV. Where, in cases I and IV, with respect to an orthonormal basis of the tangent space of $M_{1}^{3}$, the matrix representation of the induced metric on $M_{1}^{3}$ is

$$
G_{1}=\left(\begin{array}{ccc}
-1 & 0 & 0 \\
0 & 1 & 0 \\
0 & 0 & 1
\end{array}\right)
$$

and the shape operator $S$ of $M_{1}^{3}$ can be put into matrix forms

$$
B_{1}=\left(\begin{array}{ccc}
\lambda_{1} & 0 & 0 \\
0 & \lambda_{2} & 0 \\
0 & 0 & \lambda_{3}
\end{array}\right) \quad \text { and } \quad B_{4}=\left(\begin{array}{ccc}
\kappa & \lambda & 0 \\
-\lambda & \kappa & 0 \\
0 & 0 & \eta
\end{array}\right), \quad(\lambda \neq 0)
$$

respectively. For cases II and III, using a pseudo-orthonormal basis of the tangent space of 
$M_{1}^{3}$, the induced metric on $M_{1}^{3}$ has matrix form

$$
G_{2}=\left(\begin{array}{ccc}
0 & 1 & 0 \\
1 & 0 & 0 \\
0 & 0 & 1
\end{array}\right)
$$

and the shape operator $S$ of $M_{1}^{3}$ can be put into matrix forms

$$
B_{2}=\left(\begin{array}{ccc}
\kappa & 0 & 0 \\
1 & \kappa & 0 \\
0 & 0 & \lambda
\end{array}\right) \quad \text { and } \quad B_{3}=\left(\begin{array}{ccc}
\kappa & 0 & 0 \\
0 & \kappa & 1 \\
-1 & 0 & \kappa
\end{array}\right) \text {, }
$$

respectively. In case IV, the matrix $B_{4}$ has two conjugate complex eigenvalues $\kappa \pm i \lambda$, but in other cases the eigenvalues of the shape operator are real numbers.

Remark 2.1. In two cases II and III, one can substitute the pseudo-orthonormal basis $\mathscr{B}:=$ $\left\{e_{1}, e_{2}, e_{3}\right\}$ by a new orthonormal basis $\tilde{\mathscr{B}}:=\left\{\tilde{e_{1}}, \tilde{e_{2}}, e_{3}\right\}$ where $\tilde{e_{1}}:=\frac{1}{2}\left(e_{1}+e_{2}\right)$ and $\tilde{e_{2}}:=\frac{1}{2}\left(e_{1}-e_{2}\right)$. Therefore, we obtain new matrix representations $\tilde{B}_{2}$ and $\tilde{B}_{3}$ (instead of $B_{2}$ and $B_{3}$, respectively) as

$$
\tilde{B_{2}}=\left(\begin{array}{ccc}
\kappa+\frac{1}{2} & \frac{1}{2} & 0 \\
-\frac{1}{2} & \kappa-\frac{1}{2} & 0 \\
0 & 0 & \lambda
\end{array}\right) \quad \text { and } \quad \tilde{B_{3}}=\left(\begin{array}{ccc}
\kappa & 0 & \frac{\sqrt{2}}{2} \\
0 & \kappa & -\sqrt{2} / 2 \\
-\frac{\sqrt{2}}{2} & -\frac{\sqrt{2}}{2} & \kappa
\end{array}\right)
$$

After this changes, to unify the notations we denote the orthonormal basis by $\mathscr{B}$ in all cases.

Notation: According to four possible matrix representations of the shape operator of $M_{1}^{3}$, we define its principal curvatures, denoted by unified notations $\kappa_{i}$ for $i=1,2,3$, as follow.

In case I, we put $\kappa_{i}:=\lambda_{i}$, for $i=1,2,3$, where $\lambda_{i}$ 's are the eigenvalues of $B_{1}$.

In cases II, where the matrix representation of $S$ is $\tilde{B_{2}}$, we take $\kappa_{i}:=\kappa$ for $i=1,2$, and $\kappa_{3}:=\lambda$.

In case III, where the shape operator has matrix representation $\tilde{B_{3}}$, we take $\kappa_{i}:=\kappa$ for $i=1,2,3$.

Finally, in the case IV, where the shape operator has matrix representation $\tilde{B}_{4}$, we put $\kappa_{1}=\kappa+i \lambda, \kappa_{2}=\kappa-i \lambda$, and $\kappa_{3}:=\eta$.

The characteristic polynomial of $S$ on $M_{1}^{3}$ is of the form $Q(t)=\prod_{i=1}^{3}\left(t-\kappa_{i}\right)=\sum_{j=0}^{3}(-1)^{j} s_{j} t^{3-j}$, where, $s_{0}:=1, s_{1}=\sum_{j=1}^{3} \kappa_{j}, s_{2}:=\sum_{1 \leq i_{1}<i_{2} \leq 3} \kappa_{i_{1}} \kappa_{i_{2}}$ and $s_{3}:=\kappa_{1} \kappa_{2} \kappa_{3}$.

For $j=1,2,3$, the $j$-th mean curvature $H_{j}$ of $M$ is defined by $H_{j}=\frac{1}{\left(\frac{3}{3}\right)} s_{j}$. When $H_{j}$ is identically null, $M_{1}^{n}$ is said to be ( $\left.j-1\right)$-minimal. 


\section{Definition 2.2.}

(i) A timelike hypersurface $x: M_{1}^{3} \rightarrow \mathbb{E}_{1}^{4}$, with diagonalizable shape operator, is said to be isoparametric if all of its principal curvatures are constant.

(ii) A timelike hypersurface $x: M_{1}^{3} \rightarrow \mathbb{E}_{1}^{4}$, with non-diagonalizable shape operator, is said to be isoparametric if the minimal polynomial its shape operator has constant coefficients.

Remark 2.3. Here we remind Theorem 4.10 from [14], which assures us that there is no isoparametric timelike hypersurface of $\mathbb{E}_{1}^{4}$ with complex principal curvatures.

The well-known Newton transformations on the hypersurface, $P_{j}: \chi(M) \rightarrow \chi(M)$, is defined by

$$
P_{0}=I, \quad P_{j}=s_{j} I-S \circ P_{j-1}, \quad(j=1,2,3),
$$

where, I is the identity map. Using its explicit formula, $P_{j}=\sum_{i=0}^{j}(-1)^{i} s_{j-i} S^{i}$ (where $S^{0}=I$ ), it can be seen that, $P_{j}$ is self-adjoint and commutative with $S$ (see [2, 17]).

Now, we define a notation as

$$
\mu_{j ; k}=\sum_{l=0}^{k}(-1)^{l}\left(\begin{array}{l}
n \\
k-l
\end{array}\right) H_{k-l} \kappa_{j}^{l} . \quad(1 \leq j \leq 3,1 \leq k<3)
$$

Corresponding to the four possible forms $\tilde{B}_{i}$ (for $1 \leq i \leq 4$ ) of $S$, the Newton transformation $P_{j}$ has different representations. In the case I, where $S_{p}=\tilde{B_{1}}$, we have $P_{j}(p)=\operatorname{diag}\left[\mu_{1 ; j}(p), \mu_{2 ; j}(p), \mu_{3 ; j}(p)\right]$, for $j=1,2$.

When $S=B_{2}$ (in the case II), we have

$$
P_{1}=\left(\begin{array}{ccc}
\kappa+\lambda-\frac{1}{2} & -\frac{1}{2} & 0 \\
\frac{1}{2} & \kappa+\lambda+\frac{1}{2} & 0 \\
0 & 0 & 2 \kappa
\end{array}\right), \quad P_{2}=\left(\begin{array}{ccc}
\left(\kappa-\frac{1}{2}\right) \lambda & -\frac{1}{2} \lambda & 0 \\
\frac{1}{2} \lambda & \left(\kappa+\frac{1}{2}\right) \lambda & 0 \\
0 & 0 & \kappa^{2}
\end{array}\right) .
$$

In the case III, we have $S_{p}=B_{3}$, and

$$
P_{1}=\left(\begin{array}{ccc}
2 \kappa & 0 & -\frac{\sqrt{2}}{2} \\
0 & 2 \kappa & \frac{\sqrt{2}}{2} \\
\frac{\sqrt{2}}{2} & \frac{\sqrt{2}}{2} & 2 \kappa
\end{array}\right), \quad P_{2}=\left(\begin{array}{ccc}
\kappa^{2}-\frac{1}{2} & -\frac{1}{2} & -\frac{\sqrt{2}}{2} \kappa \\
\frac{1}{2} & \kappa^{2}+\frac{1}{2} & \frac{\sqrt{2}}{2} \kappa \\
\frac{\sqrt{2}}{2} \kappa & \frac{\sqrt{2}}{2} \kappa & \kappa^{2}
\end{array}\right) .
$$

In the case IV, $S=B_{4}$,

$$
P_{1}=\left(\begin{array}{ccc}
\kappa+\eta & -\lambda & 0 \\
\lambda & \kappa+\eta & 0 \\
0 & 0 & 2 \kappa
\end{array}\right), \quad P_{2}=\left(\begin{array}{ccc}
\kappa \eta & -\lambda \eta & 0 \\
\lambda \eta & \kappa \eta & 0 \\
0 & 0 & \kappa^{2}+\lambda^{2}
\end{array}\right)
$$


Fortunately, in all cases we have the following important identities for $j=1,2$, similar to those in $[2,17]$.

$$
\begin{aligned}
& \operatorname{tr}\left(P_{1}\right)=6 H_{1}, \quad \operatorname{tr}\left(P_{2}\right)=3 H_{2}, \quad \operatorname{tr}\left(P_{1} \circ S\right)=6 H_{2}, \quad \operatorname{tr}\left(P_{2} \circ S\right)=3 H_{3}, \\
& \operatorname{tr} S^{2}=9 H_{1}^{2}-6 H_{2}, \quad \operatorname{tr}\left(P_{1} \circ S^{2}\right)=9 H_{1} H_{2}-3 H_{3}, \quad \operatorname{tr}\left(P_{2} \circ S^{2}\right)=3 H_{1} H_{2} .
\end{aligned}
$$

The linearized operator of the $(j+1)$ th mean curvature of $M, L_{j}: \mathscr{C}^{\infty}(M) \rightarrow \mathscr{C}^{\infty}(M)$ is defined by the formula $L_{j}(f):=\operatorname{tr}\left(P_{j} \circ \nabla^{2} f\right)$, where, $\left\langle\nabla^{2} f(X), Y>=<\nabla_{X} \nabla f, Y>\right.$ for every $X, Y \in$ $\chi(M)$.

Associated to the orthonormal frame $\left\{e_{1}, e_{2}, e_{3}\right\}$ of tangent space on a local coordinate system in the hypersurface $x: M_{1}^{3} \rightarrow \mathbb{E}_{1}^{4}, L_{1}(f)$ has an explicit expression as

$$
L_{1}(f)=\sum_{i=1}^{3} \epsilon_{i} \mu_{i, 1}\left(e_{i} e_{i} f-\nabla_{e_{i}} e_{i} f\right) .
$$

For a Lorentzian hypersurface $x: M_{1}^{3} \rightarrow \mathbb{E}_{1}^{4}$, with a chosen (local) unit normal vector field $\mathbf{n}$, for an arbitrary vector $\mathbf{a} \in \mathbb{E}_{1}^{4}$ we use the decomposition $\mathbf{a}=\mathbf{a}^{T}+\mathbf{a}^{N}$ where $\mathbf{a}^{T} \in T M$ is the tangential component of $\mathbf{a}, \mathbf{a}^{N} \perp T M$, and we have the following formulae from [2, 17].

$$
\begin{gathered}
\nabla<x, \mathbf{a}>=\mathbf{a}^{T}, \quad \nabla<\mathbf{n}, \mathbf{a}>=-S \mathbf{a}^{T} . \\
L_{1} x=c_{1} H_{2} \mathbf{n}, \quad L_{1} \mathbf{n}=-3 \nabla\left(H_{2}\right)-3\left[3 H_{1} H_{2}-H_{3}\right] \mathbf{n}, \\
L_{1}^{2} x=6 L_{1}\left(H_{2} \mathbf{n}\right)=-6\left[9 H_{2} \nabla H_{2}-2 P_{2} \nabla H_{2}\right]-6\left[9 H_{1} H_{2}^{2}+3 H_{2} H_{3}-L_{1} H_{2}\right] \mathbf{n} .
\end{gathered}
$$

Assume that a hypersurface $x: M_{1}^{3} \rightarrow \mathbb{E}_{1}^{4}$ satisfies the condition $L_{1}^{2} x=0$, then it is said to be $L_{1}$ biharmonic. An $L_{1}$-biharmonic hypersurface $x: M_{1}^{3} \rightarrow \mathbb{E}_{1}^{4}$ is said to be proper- $L_{1}$-biharmonic, if it satisfies the condition $L_{1} x \neq 0$.

By equalities (2.7) and (2.8), from the condition $L_{1}\left(H_{2} \mathbf{n}\right)=0$ (which is equivalent to $L_{1}$ biharmonicity) we obtain simpler conditions on $M_{1}^{3}$ to be a $L_{1}$-biharmonic hypersurface in $\mathbb{E}_{1}^{4}$, as:

$$
\text { (i) } L_{1} H_{2}=3\left(3 H_{1} H_{2}^{2}-H_{2} H_{3}\right)=H_{2} \operatorname{tr}\left(S^{2} \circ P_{1}\right), \quad \text { (ii) } P_{2} \nabla H_{2}=\frac{9}{2} H_{2} \nabla H_{2} \text {. }
$$

The structure equations of $\mathbb{E}_{1}^{4}$ are given by

$$
\begin{aligned}
d \omega_{i} & =\sum_{j=1}^{4} \omega_{i j} \wedge \omega_{j}, \quad \omega_{i j}+\omega_{j i}=0, \\
d \omega_{i j} & =\sum_{l=1}^{4} \omega_{i l} \wedge \omega_{l j} .
\end{aligned}
$$

With restriction to $M$, we have $\omega_{4}=0$ and then,

$$
0=d \omega_{4}=\sum_{i=1}^{3} \omega_{4, i} \wedge \omega_{i} .
$$


By Cartan's lemma, there exist functions $h_{i j}$ such that

$$
\omega_{4, i}=\sum_{j=1}^{3} h_{i j} \omega_{j}, \quad h_{i j}=h_{j i} .
$$

This gives the second fundamental form of $M$, as $B=\sum_{i, j} h_{i j} \omega_{i} \omega_{j} e_{4}$. The mean curvature $H$ is given by $H=\frac{1}{3} \sum_{i=1}^{3} h_{i i}$. From (2.10)-(2.13) we obtain the structure equations of $M$ as follow.

$$
\begin{aligned}
d \omega_{i} & =\sum_{j=1}^{3} \omega_{i j} \wedge \omega_{j}, \quad \omega_{i j}+\omega_{j i}=0, \\
d \omega_{i j} & =\sum_{k=1}^{3} \omega_{i k} \wedge \omega_{k j}-\frac{1}{2} \sum_{k, l=1}^{3} R_{i j k l} \omega_{k} \wedge \omega_{l},
\end{aligned}
$$

for $i, j=1,2,3$, and the Gauss equations

$$
R_{i j k l}=\left(h_{i k} h_{j l}-h_{i l} h_{j k}\right)
$$

where $R_{i j k l}$ denotes the components of the Riemannian curvature tensor of $M$.

Let $h_{i j k}$ denote the covariant derivative of $h_{i j}$. We have

$$
d h_{i j}=\sum_{k=1}^{3} h_{i j k} \omega_{k}+\sum_{k=1}^{3} h_{k j} \omega_{i k}+\sum_{k=1}^{3} h_{i k} \omega_{j k} .
$$

Thus, by exterior differentiation of (2.13), we obtain the Codazzi equation

$$
h_{i j k}=h_{i k j}
$$

Now we recall the definition of an $L_{1}$-finite type hypersurface from [12], which is the basic notion of the paper.

Definition 2.4. An isometrically immersed hypersurface $x: M_{1}^{3} \rightarrow \mathbb{E}_{1}^{4}$ is said to be of $L_{1}$-finite type if $x$ has a finite decomposition $x=\sum_{i=0}^{m} x_{i}$, for some positive integer $m$, satisfying the condition $L_{1} x_{i}=\tau_{i} x_{i}$, where, $\tau_{i} \in \mathbb{R}$ and $x_{i}: M^{3} \rightarrow \mathbb{E}_{1}^{4}$ is smooth maps, for $i=1,2, \ldots, m$, and $x_{0}$ is constant. If all $\tau_{i}$ 's are mutually different, $M^{n}$ is said to be of $L_{1}-m$-type. An $L_{1}-m$-type hypersurface is said to be null if for at least one $i(1 \leq i \leq m)$ we have $\tau_{i}=0$.

\section{3. $L_{1}$-biharmonic timelike hypersurfeces of $\mathbb{E}_{1}^{4}$}

The next lemma can be proved by a similar proof as in [20]. 
Lemma 3.1. Let $M_{1}^{3}$ be a timelike hypersurface in $\mathbb{E}_{1}^{4}$ of type I with principal curvatures of constant multiplicities. Then the distribution of the space of principal directions corresponding to the principal curvatures is completely integrable. In addition, if a principal curvature is of multiplicity greater than one, then it will be constant on each integral submanifold of the corresponding distribution.

Theorem 3.2. There is no $L_{1}$-biharmonic $L_{1}$-finite type Lorentzian hypersurface in $\mathbb{E}_{1}^{4}$.

Proof. Let $x: M_{1}^{3} \rightarrow \mathbb{E}_{1}^{4}$ be an $L_{1}$-biharmonic $L_{1}$-finite type Lorentzian hypersurface in $\mathbb{E}_{1}^{4}$. By assumption, the map $x$ has a finite decomposition as

$$
x=x_{0}+x_{t_{1}}+\cdots+x_{t_{k}}
$$

with $L_{1} x_{0}=0$ and $L_{1} x_{t_{i}}=\lambda_{t_{i}} x_{t_{i}}$ for nonzero distinct eigenvalues $\lambda_{t_{1}}, \ldots, \lambda_{t_{k}}$ of $L_{1}$. By the action of $L_{1}^{S}$ on both sides of equality (3.1), we obtain

$$
0=L_{1}^{s} x=\lambda_{t_{1}}^{s} x_{t_{1}}+\cdots+\lambda_{t_{k}}^{s} x_{t_{k}}
$$

for $s=1,2,3, \ldots$. Since $\lambda_{t_{1}}, \ldots, \lambda_{t_{k}}$ are distinct eigenvalues of $L_{1}$, equation (3.2) can not hold.

Corollary 3.3. There is no $L_{1}$-biharmonic $L_{1}$-finite type connected orientable Riemannian or Lorentzian hypersurface in the Lorentz-Minkowski 4-space.

For instance, we see two examples of non $L_{1}$-biharmonic timelike hypersurfaces in $\mathbb{E}_{1}^{4}$.

Example 3.4. Let $M_{1}^{3}(r)$ be the product $\mathbb{S}_{1}^{2}(r) \times \mathbb{E}^{1} \subset \mathbb{E}_{1}^{4}$ where $r>0$. It has another representation as

$$
M_{1}^{3}(r)=\left\{\left(y_{1}, \ldots, y_{4}\right) \in \mathbb{E}_{1}^{4} \mid-y_{1}^{2}+y_{2}^{2}+y_{3}^{2}=r^{2}\right\},
$$

having the spacelike vector field $\mathbf{n}(y)=-\frac{1}{r}\left(y_{1}, y_{2}, y_{3}, 0\right)$ as the Gauss map. Clearly, it has two distinct principal curvatures $\kappa_{1}=\kappa_{2}=\frac{1}{r}, \kappa_{3}=0$, and the constant higher order mean curvatures $H_{1}=\frac{2}{3} r^{-1}, H_{2}=\frac{1}{3} r^{-2}$ and $H_{3}=0$. One can see that $L_{1}^{2} x \neq 0$.

Example 3.5. Let $\bar{M}_{1}^{3}(r)$ be the product $\mathbb{E}_{1}^{2} \times \mathbb{S}^{1}(r) \subset \mathbb{E}_{1}^{4}$ where $r>0$. It can be represented as

$$
\bar{M}_{1}^{3}(r)=\left\{\left(y_{1}, \ldots, y_{4}\right) \in \mathbb{R}_{1}^{4} \mid y_{3}^{2}+y_{4}^{2}=r^{2}\right\}
$$

with the Gauss map $\mathbf{n}(y)=-\frac{1}{r}\left(0,0, y_{3}, y_{4}\right)$. it has two distinct principal curvatures $\kappa_{1}=\kappa_{2}=0$, $\kappa_{3}=\frac{1}{r}$, and the constant higher order mean curvatures $H_{1}=\frac{1}{3 r}$, and $H_{k}=0$ for $k=2,3$. So, Also, one can see that $L_{1}^{2} x \neq 0$ we have $L_{k}^{2} x=0$ for $k=2,3$. 


\subsection{Timelike hypersurfeces of type I}

Proposition 3.6. Let $x: M_{1}^{3} \rightarrow \mathbb{E}_{1}^{4}$ be an $L_{1}$-biharmonic connected orientable timelike hypersurface with three distinct real principal curvatures and constant ordinary mean curvature. Then, the 2nd mean curvature of $M$ has to be constant.

Proof. Suppose that, $H_{2}$ is non-constant. Considering the open subset $\mathscr{U}=\left\{p \in M: \nabla H_{2}^{2}(p) \neq\right.$ $0\}$, we try to show $\mathscr{U}=\varnothing$. By the assumption $M_{1}^{3}$ has three distinct principal curvature, then, with respect to a suitable (local) orthonormal tangent frame $\left\{e_{1}, e_{2}, e_{3}\right\}$ on $M$, the shape operator $S$ has the matrix form $B_{1}$, such that $S e_{i}=\lambda_{i} e_{i}$ and then, $P_{2} e_{i}=\mu_{i, 2} e_{i}$ for $i=1,2,3$. Using the polar decomposition $\nabla H_{2}=\sum_{i=1}^{3} \epsilon_{i} e_{i}\left(H_{2}\right) e_{i}$, from condition (2.9(ii)) we get

$$
e_{i}\left(H_{2}\right)\left(\mu_{i, 2}-\frac{9}{2} H_{2}\right)=0
$$

for $i=1,2,3$. Each point of $\mathscr{U}$ has an open neighborhood on which we have $e_{i}\left(H_{2}\right) \neq 0$ for at least one $i$. So, without loss of generality, we can assume that $e_{1}\left(H_{2}\right) \neq 0$ and then we have $\mu_{1,2}=\frac{9}{2} H_{2}$, (locally) on $\mathscr{U}$, which gives $\lambda_{2} \lambda_{3}=\frac{9}{2} H_{2}$. Now, we prove three simple claims.

Claim 1: $e_{2}\left(H_{2}\right)=e_{3}\left(H_{2}\right)=0$.

If $e_{2}\left(H_{2}\right) \neq 0$ or $e_{3}\left(H_{2}\right) \neq 0$, then by (3.3) we get $\mu_{1,2}=\mu_{2,2}=\frac{9}{2} H_{2}$ or $\mu_{1,2}=\mu_{3,2}=\frac{9}{2} H_{2}$, which give $\lambda_{3}\left(\lambda_{2}-\lambda_{1}\right)=0$ or $\lambda_{2}\left(\lambda_{1}-\lambda_{3}\right)=0$. But, since $\lambda_{i}$ 's are assumed to be mutually distinct, we get $\lambda_{3}=0$ or $\lambda_{2}=0$, which gives $H_{2}=0$ on $\mathscr{U}$. The result is in contradiction with the definition of $\mathscr{U}$.

Claim 2: $e_{2}\left(\lambda_{1}\right)=e_{3}\left(\lambda_{1}\right)=0$.

Since $H$ is assumed to be constant on $M$, we have $e_{2}\left(\lambda_{1}\right)=e_{2}\left(3 H-\lambda_{1}-\lambda_{2}\right)=-e_{2}\left(\lambda_{1}\right)-e_{2}\left(\lambda_{2}\right)$. On the other hand, from two recent results $e_{2}\left(H_{2}\right)=0$ and $\lambda_{2} \lambda_{3}=\frac{9}{2} H_{2}$ we get

$$
e_{2}\left(\lambda_{1} \lambda_{3}\right)+e_{2}\left(\lambda_{1} \lambda_{2}\right)=e_{2}\left(3 H_{2}-\frac{9}{2} H_{2}\right)=0,
$$

which gives $\lambda_{1} e_{2}\left(\lambda_{2}+\lambda_{3}\right)+\left(\lambda_{2}+\lambda_{3}\right) e_{2} \lambda_{1}=0$, and then we have

$$
\lambda_{1} e_{2}\left(3 H-\lambda_{1}\right)+\left(\lambda_{2}+\lambda_{3}\right) e_{2} \lambda_{1}=\lambda_{1} e_{2}\left(-\lambda_{1}\right)+\left(\lambda_{2}+\lambda_{3}\right) e_{2} \lambda_{1}=\left(-\lambda_{1}+\lambda_{2}+\lambda_{3}\right) e_{2} \lambda_{1}=0
$$

Therefore, assuming $e_{2}\left(\lambda_{1}\right) \neq 0$, we get $\lambda_{1}=\lambda_{2}+\lambda_{3}$ which gives contradiction

$$
e_{2}\left(\lambda_{1}\right)=e_{2}\left(\lambda_{2}+\lambda_{3}\right)=e_{2}\left(3 H-\lambda_{1}\right)=-e_{2}\left(\lambda_{1}\right)
$$

Consequently, $e_{2}\left(\lambda_{1}\right)=0$.

Similarly, one can show $e_{3}\left(\lambda_{1}\right)=0$. So, Claim 2 is proved. 
Claim 3: $e_{2}\left(\lambda_{3}\right)=e_{3}\left(\lambda_{2}\right)=0$.

Using the notations

$$
\nabla_{e_{i}} e_{j}=\sum_{k=1}^{3} \omega_{i j}^{k} e_{k}, \quad(i, j=1,2,3),
$$

and the compatibility condition $\nabla_{e_{k}}<e_{i}, e_{j}>=0$, we have

$$
\omega_{k i}^{i}=0, \quad \omega_{k i}^{j}+\omega_{k j}^{i}=0, \quad(i, j, k=1,2,3)
$$

and applying the Codazzi equation (see [16], page 115, Corollary 34(2))

$$
\left.\left(\nabla_{V} S\right) W=\nabla_{W} S\right) V, \quad(\forall V, W \in \chi(M))
$$

on the basis $\left\{e_{1}, e_{2}, e_{3}\right\}$, we get for distinct $i, j, k=1,2,3$

$$
\text { (a) } e_{i}\left(\lambda_{j}\right)=\left(\lambda_{i}-\lambda_{j}\right) \omega_{j i}^{j}, \quad \text { (b) }\left(\lambda_{i}-\lambda_{j}\right) \omega_{k i}^{j}=\left(\lambda_{k}-\lambda_{j}\right) \omega_{i k}^{j} \text {. }
$$

Also, by a straightforward computation of components of the identity $\left.\left.\nabla_{e_{i}} S\right) e_{j}-\nabla_{e_{j}} S\right) e_{i} \equiv 0$ for distinct $i, j=1,2,3$, we get $\left[e_{2}, e_{3}\right]\left(H_{2}\right)=0, \omega_{12}^{1}=\omega_{13}^{1}=\omega_{13}^{2}=\omega_{21}^{3}=\omega_{32}^{1}=0$ and

$$
\omega_{21}^{2}=\frac{e_{1}\left(\lambda_{2}\right)}{\lambda_{1}-\lambda_{2}}, \quad \omega_{31}^{3}=\frac{e_{1}\left(\lambda_{3}\right)}{\lambda_{1}-\lambda_{3}}, \omega_{23}^{2}=\frac{e_{3}\left(\lambda_{2}\right)}{\lambda_{3}-\lambda_{2}}, \quad \omega_{32}^{3}=\frac{e_{2}\left(\lambda_{3}\right)}{\lambda_{2}-\lambda_{3}} .
$$

Therefore, the covariant derivatives $\nabla_{e_{i}} e_{j}$ simplify to $\nabla_{e_{1}} e_{k}=0$ for $k=1,2,3$, and

$$
\begin{array}{lll}
\nabla_{e_{2}} e_{1}=\frac{e_{1}\left(\lambda_{2}\right)}{\lambda_{1}-\lambda_{2}} e_{2}, & \nabla_{e_{3}} e_{1}=\frac{e_{1}\left(\lambda_{3}\right)}{\lambda_{1}-\lambda_{3}} e_{3}, & \nabla_{e_{2}} e_{2}=\frac{e_{1}\left(\lambda_{2}\right)}{\lambda_{2}-\lambda_{1}} e_{1}, \\
\nabla_{e_{3}} e_{2}=\frac{e_{2}\left(\lambda_{3}\right)}{\lambda_{2}-\lambda_{3}} e_{3}, & \nabla_{e_{2}} e_{3}=\frac{e_{3}\left(\lambda_{2}\right)}{\lambda_{3}-\lambda_{2}} e_{2}, & \nabla_{e_{3}} e_{3}=\frac{e_{1}\left(\lambda_{3}\right)}{\lambda_{3}-\lambda_{1}} e_{1}+\frac{e_{2}\left(\lambda_{3}\right)}{\lambda_{3}-\lambda_{2}} e_{2} .
\end{array}
$$

Now, the Gauss equation for $<R\left(e_{2}, e_{3}\right) e_{1}, e_{2}>$ and $<R\left(e_{2}, e_{3}\right) e_{1}, e_{3}>$ show that

$$
\begin{aligned}
& e_{3}\left(\frac{e_{1}\left(\lambda_{2}\right)}{\lambda_{1}-\lambda_{2}}\right)=\frac{e_{3}\left(\lambda_{2}\right)}{\lambda_{3}-\lambda_{2}}\left(\frac{e_{1}\left(\lambda_{3}\right)}{\lambda_{1}-\lambda_{3}}-\frac{e_{1}\left(\lambda_{2}\right)}{\lambda_{1}-\lambda_{2}}\right), \\
& e_{2}\left(\frac{e_{1}\left(\lambda_{3}\right)}{\lambda_{1}-\lambda_{3}}\right)=\frac{e_{2}\left(\lambda_{3}\right)}{\lambda_{2}-\lambda_{3}}\left(\frac{e_{1}\left(\lambda_{3}\right)}{\lambda_{1}-\lambda_{3}}-\frac{e_{1}\left(\lambda_{2}\right)}{\lambda_{1}-\lambda_{2}}\right) .
\end{aligned}
$$

We also have the Gauss equation for $<R\left(e_{1}, e_{2}\right) e_{1}, e_{2}>$ and $<R\left(e_{3}, e_{1}\right) e_{1}, e_{3}>$, which give the following relations

$$
e_{1}\left(\frac{e_{1}\left(\lambda_{2}\right)}{\lambda_{1}-\lambda_{2}}\right)+\left(\frac{e_{1}\left(\lambda_{2}\right)}{\lambda_{1}-\lambda_{2}}\right)^{2}=\lambda_{1} \lambda_{2}, \quad e_{1}\left(\frac{e_{1}\left(\lambda_{3}\right)}{\lambda_{1}-\lambda_{3}}\right)+\left(\frac{e_{1}\left(\lambda_{3}\right)}{\lambda_{3}-\lambda_{1}}\right)^{2}=\lambda_{1} \lambda_{3} .
$$

Finally, we obtain from the Gauss equation for $<R\left(e_{3}, e_{1}\right) e_{2}, e_{3}>$ that

$$
e_{1}\left(\frac{e_{2}\left(\lambda_{3}\right)}{\lambda_{2}-\lambda_{3}}\right)=\frac{e_{1}\left(\lambda_{3}\right) e_{2}\left(\lambda_{3}\right)}{\left(\lambda_{3}-\lambda_{1}\right)\left(\lambda_{2}-\lambda_{3}\right)}
$$


On the other hand, we consider the $L_{1}$-biharmonic condition (2.9). It follows from Claim I that

$$
-\mu_{1,1} e_{1} e_{1}\left(H_{2}\right)+\left(\mu_{2,1} \frac{e_{1}\left(\lambda_{2}\right)}{\lambda_{2}-\lambda_{1}}+\mu_{3,1} \frac{e_{1}\left(\lambda_{3}\right)}{\lambda_{3}-\lambda_{1}}\right) e_{1}\left(H_{2}\right)-9 H_{2}^{2}\left(H-\frac{3}{2} \lambda_{1}\right)=0 .
$$

By differentiating (3.14) along on $e_{2}$ respectively $e_{3}$, and using (3.10), (3.11) we obtain

$$
\begin{aligned}
& e_{2}\left(\frac{e_{1}\left(\lambda_{2}\right)}{\lambda_{2}-\lambda_{1}}\right)=\frac{e_{2}\left(\lambda_{3}\right)}{\lambda_{2}-\lambda_{3}}\left(\frac{e_{1}\left(\lambda_{3}\right)}{\lambda_{1}-\lambda_{3}}-\frac{e_{1}\left(\lambda_{2}\right)}{\lambda_{1}-\lambda_{2}}\right), \\
& e_{3}\left(\frac{e_{1}\left(\lambda_{3}\right)}{\lambda_{3}-\lambda_{1}}\right)=\frac{e_{3}\left(\lambda_{2}\right)}{\lambda_{3}-\lambda_{2}}\left(\frac{e_{1}\left(\lambda_{2}\right)}{\lambda_{1}-\lambda_{2}}-\frac{e_{1}\left(\lambda_{3}\right)}{\lambda_{1}-\lambda_{3}}\right) .
\end{aligned}
$$

Using (3.9), we find that

$$
\left[e_{1}, e_{2}\right]=\frac{e_{1}\left(\lambda_{2}\right)}{\lambda_{2}-\lambda_{1}} e_{2}
$$

Applying both sides of the equality (3.17) on $\frac{e_{1}\left(\lambda_{2}\right)}{\lambda_{2}-\lambda_{1}}$, using (3.15), (3.12), and (3.13), we deduce that

$$
\frac{e_{2}\left(\lambda_{3}\right)}{\lambda_{2}-\lambda_{3}}\left(\frac{e_{1}\left(\lambda_{3}\right)}{\lambda_{3}-\lambda_{1}}+\frac{e_{1}\left(\lambda_{2}\right)}{\lambda_{1}-\lambda_{2}}\right)=0 .
$$

(3.18) shows that $e_{2}\left(\lambda_{3}\right)=0$ or

$$
\frac{e_{1}\left(\lambda_{3}\right)}{\lambda_{3}-\lambda_{1}}=\frac{e_{1}\left(\lambda_{2}\right)}{\lambda_{2}-\lambda_{1}}
$$

From equation (3.19), by differentiating on its both sides along $e_{1}$ and applying (3.12), we get $\lambda_{2}=\lambda_{3}$, which is a contradiction, so we have to confirm the result $e_{2}\left(\lambda_{3}\right)=0$.

Analogously, using (3.9), we find that $\left[e_{1}, e_{3}\right]=\frac{e_{1}\left(\lambda_{3}\right)}{\lambda_{3}-\lambda_{1}} e_{3}$. By a similar manner, we deduce that

$$
\frac{e_{3}\left(\lambda_{2}\right)}{\lambda_{3}-\lambda_{2}}\left(\frac{e_{1}\left(\lambda_{2}\right)}{\lambda_{2}-\lambda_{1}}+\frac{e_{1}\left(\lambda_{3}\right)}{\lambda_{1}-\lambda_{3}}\right)=0,
$$

and one can show that $e_{3}\left(\lambda_{2}\right)$ necessarily has to be vanished.

Hence, we have obtained $e_{2}\left(\lambda_{3}\right)=e_{3}\left(\lambda_{2}\right)=0$ which, by applying the Gauss equation for $<R\left(e_{2}, e_{3}\right) e_{1}, e_{3}>$, gives the following equality

$$
\frac{e_{1}\left(\lambda_{3}\right) e_{1}\left(\lambda_{2}\right)}{\left(\lambda_{3}-\lambda_{1}\right)\left(\lambda_{2}-\lambda_{1}\right)}=\lambda_{2} \lambda_{3}
$$

Finally, using (3.12), differentiating (3.21) along $e_{1}$ gives

$$
\lambda_{2} \lambda_{3}\left(\frac{e_{1}\left(\lambda_{3}\right)}{\lambda_{3}-\lambda_{1}}+\frac{e_{1}\left(\lambda_{2}\right)}{\lambda_{1}-\lambda_{2}}\right)=0
$$

which implies $\lambda_{2} \lambda_{3}=0$ (since we have seen above that $\left(\frac{e_{1}\left(\lambda_{3}\right)}{\lambda_{3}-\lambda_{1}}+\frac{e_{1}\left(\lambda_{2}\right)}{\lambda_{1}-\lambda_{2}}\right) \neq 0$ ). Therefore, we obtain $H_{2}=0$ on $\mathscr{U}$, which is a contradiction. Hence $H_{2}$ is constant on $M^{3}$. 
Theorem 3.7. Let $x: M_{1}^{3} \rightarrow \mathbb{E}_{1}^{4}$ be an $L_{1}$-biharmonic timlike hypersurface with shape operator of type I in $\mathbb{E}_{1}^{4}$. If $M_{1}^{3}$ has constant mean curvature and three distinct principal curvatures, then it is 1-minimal.

Proof. By assumption $H_{1}$ is assumed to be constant and then, by Theorem 3.6 it is proved that $\mathrm{H}_{2}$ has to be constant. We claim that $H_{2}$ is null. If $H_{2} \neq 0$, by using [(2.9)(i)] we obtain that $H_{3}$ is constant. Therefore, all of mean curvatures $H_{i}$ (for $i=1,2,3$ ) are constant, which means that $M^{3}$ is isoparametric. By Corollary 2.7 in [14], an isoparametric Lorentzian hypersurface of Case I in the Einstein space has at most one nonzero principal curvature distinct principal, which contradicts with the assumption that, three principal curvatures of $M$ are assumed to be mutually distinct. So $\mathrm{H}_{2} \equiv 0$.

Theorem 3.8. Let $x: M_{1}^{3} \rightarrow \mathbb{E}_{1}^{4}$ be a $L_{1}$-biharmonic Lorentzian hypersurfaces of $\mathbb{E}_{1}^{4}$ with diagonalizable shape operator (i.e of type I). If $M_{1}^{3}$ has exactly two distinct principal curvatures, then it is 1-minimal.

Proof. By assumption, $M_{1}^{3}$ has two distinct principal curvatures $\lambda_{1}$ and $\lambda_{2}$ of multiplicities 1 and 2, respectively. Defining the open subset $\mathscr{U}$ of $M$ as $\mathscr{U}:=\left\{p \in M_{1}^{3}: \nabla H_{2}^{2}(p) \neq 0\right\}$, we prove that $\mathscr{U}$ is empty. Assuming $\mathscr{U} \neq \varnothing$, we consider $\left\{e_{1}, e_{2}, e_{3}\right\}$ as a local orthonormal frame of principal directions of $S$ on $\mathscr{U}$ such that $S e_{i}=\lambda_{i} e_{i}$ for $i=1,2,3$. By assumption, we have

$$
\lambda_{1}=\lambda_{2}=\lambda, \quad \lambda_{3}=\mu \text {. }
$$

Therefore, we obtain

$$
\mu_{1,2}=\mu_{2,2}=\lambda \mu, \quad \mu_{3,2}=\lambda^{2}, \quad 3 H_{2}=\lambda^{2}+2 \lambda \mu .
$$

By condition [(2.9) (ii)], we have

$$
P_{2}\left(\nabla H_{2}\right)=\frac{9}{2} H_{2} \nabla H_{2}
$$

Then, using the polar decomposition

$$
\nabla H_{2}=\sum_{i=1}^{3} \epsilon_{i}<\nabla H_{2}, e_{i}>e_{i},
$$

we see that (3.24) is equivalent to

$$
\epsilon_{i}<\nabla H_{2}, e_{i}>\left(\mu_{i, 2}-\frac{9}{2} H_{2}\right)=0
$$

on $\mathscr{U}$ for $i=1,2,3$. Hence, for every $i$ such that $<\nabla H_{2}, e_{i}>\neq 0$ on $\mathscr{U}$ we get

$$
\mu_{i, 2}=\frac{9}{2} H_{2} .
$$


By definition, we have $\nabla H_{2} \neq 0$ on $\mathscr{U}$, which gives one or both of the following states.

State 1. $<\nabla H_{2}, e_{i}>\neq 0$, for $i=1$ or $i=2$. By equalities (3.23) and (3.26), we obtain

$$
\lambda \mu=\frac{9}{2}\left(\frac{2}{3} \lambda \mu+\frac{1}{3} \lambda^{2}\right)
$$

which gives

$$
\lambda\left(2 \mu+\frac{3}{2} \lambda\right)=0
$$

If $\lambda=0$ then $H_{2}=0$. Otherwise, we get $\mu=-\frac{3}{4} \lambda, H_{2}=-\frac{1}{6} \lambda^{2}$.

State 2. $<\nabla H_{2}, e_{3}>\neq 0$. By equalities (3.23) and (3.26), we obtain

$$
\lambda^{2}=\frac{9}{2}\left(\frac{2}{3} \lambda \mu+\frac{1}{3} \lambda^{2}\right)
$$

which gives

$$
\lambda\left(3 \mu+\frac{1}{2} \lambda\right)=0 .
$$

If $\lambda=0$ then $H_{2}=0$. Otherwise, we have $\mu=-\frac{1}{6} \lambda, H_{2}=\frac{2}{9} \lambda^{2}$.

Both states requires the same calculation, so we consider for instance State 2.

By Lemma 3.1, let us denote the maximal integral submanifold through $x \in \mathscr{U}$, corresponding to $\lambda$ by $\mathscr{U}_{1}^{n-1}(x)$. We write

$$
d \lambda=\sum_{i=1}^{3} \lambda_{, i} \omega_{i} \quad d \mu=\sum_{j=1}^{3} \mu_{, j} \omega_{j} .
$$

Then, Lemma 3.1 implies that $\lambda_{, 1}=\lambda_{, 2}=0$. We can assume that $\lambda>0$ on $\mathscr{U}$, then we have (in State 2)

$$
\mu=\frac{-1}{6} \lambda<0
$$

By means of (2.17), we obtain

$$
\sum_{k=1}^{3} h_{i j k} \omega_{k}=\delta_{i j} d \lambda_{j}+\left(\lambda_{i}-\lambda_{j}\right) \omega_{i j},
$$

for $i, j, k=1,2,3$. Here, we adopt the notational convention that $a, b, c=1,2$.

From (3.29) and (3.31), we have

$$
\begin{aligned}
& h_{12 k}=h_{21 k}=0, \\
& h_{a a b}=0, \quad h_{a a 3}=\lambda_{, 3}, \\
& h_{33 a}=0, \quad h_{333}=\mu_{, 3} .
\end{aligned}
$$


Combining this with (2.18) and the formula

$$
\sum_{i=1}^{3} h_{a 3 i} \omega_{i}=d h_{a 3}+\sum_{i=1}^{3} h_{i 3} \omega_{i a}+\sum_{i=1}^{3} h_{a i} \omega_{i 3}=(\lambda-\mu) \omega_{a 3},
$$

we obtain from (3.30)

$$
\omega_{a 3}=\frac{\lambda, 3}{\lambda-\mu} \omega_{a}=\frac{6 \lambda, 3}{7 \lambda} \omega_{a} .
$$

Therefore we have

$$
d \omega_{3}=\sum_{a=1}^{2} \omega_{3 a} \wedge \omega_{a}=0 .
$$

Notice that we may consider $\lambda$ to be locally a function of the parameter $s$, where $s$ is the arc length of an orthogonal trajectory of the family of the integral submanifolds corresponding to $\lambda$. We may put $\omega_{3}=d s$.

Thus, for $\lambda=\lambda(s)$, we have

$$
d \lambda=\lambda_{, 3} d s, \quad \lambda_{, 3}=\lambda^{\prime}(s)
$$

so from (3.33), we get

$$
\omega_{a 3}=\frac{\lambda_{, 3}}{\lambda-\mu} \omega_{a}=\frac{6 \lambda^{\prime}(s)}{7 \lambda} \omega_{a} .
$$

According to the structure equations of $\mathbb{E}_{1}^{4}$ and (3.34), we may compute

$$
\text { (i): } \begin{aligned}
d \omega_{a 3} & =\sum_{b=1}^{2} \omega_{a b} \wedge \omega_{b 3}+\omega_{a 4} \wedge \omega_{43}=\left(\frac{6 \lambda^{\prime}}{7 \lambda}\right) \sum_{b=1}^{2} \omega_{a b} \wedge \omega_{b}-\lambda \mu \omega_{a} \wedge d s, \\
& \left.=\left\{-\left(\frac{6 \lambda_{a 3}}{7 \lambda}\right)^{\prime}+\left(\frac{6 \lambda^{\prime}}{7 \lambda}\right)^{2}\right\} \omega_{a} \wedge d s+\left(\frac{6 \lambda^{\prime}}{7 \lambda}\right)^{\prime} d s \wedge \omega_{a}+\left(\frac{6 \lambda^{\prime}}{7 \lambda}\right) d \omega_{a}\right) \sum_{b=1}^{2} \omega_{a b} \wedge \omega_{b} .
\end{aligned}
$$

Comparing equalities [(3.35)(i)] and [(3.35)(ii)], we get $\left(\frac{6 \lambda^{\prime}}{7 \lambda}\right)^{\prime}-\left(\frac{6 \lambda^{\prime}}{7 \lambda}\right)^{2}-\lambda \mu=0$, which, by combining with (3.30), gives

$$
\left(\frac{6 \lambda^{\prime}}{7 \lambda}\right)^{\prime}-\left(\frac{6 \lambda^{\prime}}{7 \lambda}\right)^{2}-\left(\frac{-1}{6}\right) \lambda^{2}=0
$$

Defining function $\beta(s):=\left(\frac{1}{\lambda(s)}\right)^{\frac{6}{7}}$ for $s \in(-\infty,+\infty)$, from (3.36) we get $\beta^{\prime \prime}=\left(\frac{1}{6}\right) \beta^{\frac{-8}{6}}$, which by integrating, gives $\left(\beta^{\prime}\right)^{2}=-\beta^{\frac{-2}{6}}+c$, where $c$ is the constant of integration. The last equation is equivalent to

$$
\left(\lambda^{\prime}\right)^{2}=-\left(\frac{7}{6}\right)^{2} \lambda^{4}+c\left(\frac{7}{6}\right)^{2} \lambda^{\frac{26}{7}}
$$


Now, in order to compare two sides of condition [(2.9)(i)], we need to compute $\nabla_{e_{i}} \nabla H_{2}$ and $P_{1}\left(e_{i}\right)$ for $i=1,2,3$. From (3.27) we have $\nabla H_{2}=\frac{4}{9} \lambda \lambda^{\prime} e_{3}$, which by using (3.34), gives

$$
\begin{aligned}
& \nabla_{e_{a}} \nabla H_{2}=\frac{4}{9} \lambda \lambda^{\prime} \nabla_{e_{a}} e_{3}=\frac{4}{9} \lambda^{r} \lambda^{\prime} \sum_{b} \omega_{3 b}\left(e_{a}\right) e_{b}=-\frac{8}{21} \lambda^{\prime 2} e_{a}, \\
& \nabla_{e_{3}} \nabla H_{2}=\frac{4}{9} \nabla_{e_{3}}\left(\lambda \lambda^{\prime} e_{3}\right)=\frac{4}{9} \lambda^{\prime 2} e_{3}+\frac{4}{9} \lambda \lambda^{\prime \prime} e_{3} .
\end{aligned}
$$

By using (3.23) and (3.30), we compute $P_{1}\left(e_{a}\right)$ and $P_{1}\left(e_{3}\right)$.

$$
P_{1}\left(e_{1}\right)=\frac{5}{6} \lambda e_{1}, \quad P_{1}\left(e_{2}\right)=\frac{5}{6} \lambda e_{2} \quad P_{1}\left(e_{3}\right)=2 \lambda e_{3} .
$$

From (3.38) and (3.39), we get

$$
L_{1} H_{2}=6 H_{2}\left(\frac{-10\left(\lambda^{\prime}\right)^{2}}{21 \lambda}+\frac{2\left(\lambda^{\prime}\right)^{2}}{3 \lambda}+\frac{2}{3} \lambda^{\prime \prime}\right) .
$$

From [(2.9)(i)], we have $L_{1} H_{2}=H_{2} \operatorname{tr}\left(S^{2} \circ P_{1}\right)=2 H_{2} \frac{11}{6} \lambda^{3}$, which Combining with (3.40), gives

$$
\lambda \lambda^{\prime \prime}+\left(1+\frac{-5}{7}\right) \lambda^{\prime 2}-2 \frac{33}{12} \lambda^{4}=0
$$

On the other hand, the equality (3.36) is equivalent to

$$
\lambda \lambda^{\prime \prime}=\frac{13}{7} \lambda^{\prime 2}+\frac{-7}{36} \lambda^{4}
$$

Now, substituting (3.42) and (3.41), we obtain

$$
\frac{15}{7} \lambda^{\prime 2}+\frac{191}{36} \lambda^{4}=0
$$

From equations (3.37), (3.43) and (3.27), we get that $H_{2}$ is locally constant on $\mathscr{U}$, which is a contradiction with the definition of $\mathscr{U}$. Hence $H_{2}$ is constant on $M$.

By a similar discussion, one can get the same result in State 1.

\subsection{Timelike hypersurfeces of types II}

Proposition 3.9. Let $x: M_{1}^{3} \rightarrow \mathbb{E}_{1}^{4}$ be an $L_{1}$-biharmonic connected orientable timelike hypersurface with shape operator of type II in $\mathbb{E}_{1}^{4}$. If $M_{1}^{3}$ has constant ordinary mean curvature, then its 2 nd mean curvature has to be constant.

Proof. Suppose that, $H_{2}$ be non-constant. Considering the open subset $\mathscr{U}=\left\{p \in M: \nabla H_{2}^{2}(p) \neq\right.$ $0\}$, we try to show $\mathscr{U}=\varnothing$. By the assumption, with respect to a suitable (local) orthonormal tangent frame $\left\{e_{1}, e_{2}, e_{3}\right\}$ on $M$, the shape operator $S$ has the matrix form $\tilde{B}_{2}$, such that $S e_{1}=\left(\kappa+\frac{1}{2}\right) e_{1}-\frac{1}{2} e_{2}, S e_{2}=\frac{1}{2} e_{1}+\left(\kappa-\frac{1}{2}\right) e_{2}, S e_{3}=\lambda e_{3}$ and then, we have $P_{2} e_{1}=\left(\kappa-\frac{1}{2}\right) \lambda e_{1}+\frac{1}{2} \lambda e_{2}$, $P_{2} e_{2}=-\frac{1}{2} \lambda e_{1}+\left(\kappa+\frac{1}{2}\right) \lambda e_{2}$ and $P_{2} e_{3}=\kappa^{2} e_{3}$. 
Using the polar decomposition $\nabla H_{2}=\sum_{i=1}^{3} \epsilon_{i} e_{i}\left(H_{2}\right) e_{i}$, from condition (2.9(ii)) we get

(i) $\epsilon_{1} e_{1}\left(H_{2}\right)\left[\left(\kappa-\frac{1}{2}\right) \lambda-\frac{9}{2} H_{2}\right]=\epsilon_{2} e_{2}\left(H_{2}\right) \frac{\lambda}{2}$

(ii) $\epsilon_{2} e_{2}\left(H_{2}\right)\left[\left(\kappa+\frac{1}{2}\right) \lambda-\frac{9}{2} H_{2}\right]=-\epsilon_{1} e_{1}\left(H_{2}\right) \frac{\lambda}{2}$,

(iii) $\epsilon_{3} e_{3}\left(H_{2}\right)\left(\kappa^{2}-\frac{9}{2} H_{2}\right)=0$.

Now, we prove some simple claims.

Claim 1: $e_{1}\left(H_{2}\right)=e_{2}\left(H_{2}\right)=e_{3}\left(H_{2}\right)=0$.

If $e_{1}\left(H_{2}\right) \neq 0$, then by dividing both sides of equalities [(3.44)(i, ii)] by $\epsilon_{1} e_{1}\left(H_{2}\right)$ we get

(i) $\left(\kappa-\frac{1}{2}\right) \lambda-\frac{9}{2} H_{2}=\frac{\epsilon_{2} e_{2}\left(H_{2}\right)}{\epsilon_{1} e_{1}\left(H_{2}\right)} \frac{\lambda}{2}$,

(ii) $\frac{\epsilon_{2} e_{2}\left(H_{2}\right)}{\epsilon_{1} e_{1}\left(H_{2}\right)}\left[\left(\kappa+\frac{1}{2}\right) \lambda-\frac{9}{2} H_{2}\right]=-\frac{\lambda}{2}$,

which, by substituting (i) in (ii), gives $\frac{\lambda}{2}(1+u)^{2}=0$, where $u:=\frac{\epsilon_{2} e_{2}\left(H_{2}\right)}{\epsilon_{1} e_{1}\left(H_{2}\right)}$. Then $\lambda=0$ or $u=-1$. If $\lambda=0$, then we get $H_{2}=0$ from [(3.45)(i)]. Also, by assumption $\lambda \neq 0$ we get $u=-1$ which gives $\kappa \lambda=\frac{9}{2} H_{2}$, then $\kappa(3 \kappa+4 \lambda)=0$ and finally $\kappa=-\frac{4}{3} \lambda$ (since $\kappa=0$ gives $H_{2}=0$ again). Hence, we have $H_{2}=\frac{2}{9} \kappa \lambda=-\frac{8}{27} \lambda^{2}$ and $H_{1}=-\frac{5}{9} \lambda$, and since $H_{1}$ is assumed to be constant, $H_{2}$ has to be constant and we have $e_{1}\left(H_{2}\right)=0$, which is a contradiction. Therefore, the first claim is proved. The second claim (i.e. $e_{2}\left(H_{2}\right)=0$ ) can be proven by a similar manner.

Now, if $e_{3}\left(H_{2}\right) \neq 0$, then by [(3.44)(iii)] we get $\kappa^{2}=\frac{9}{2} H_{2}$, then $\kappa(\kappa+6 \lambda)=0$, which gives $\kappa=0$ or $\kappa=-6 \lambda$. If $\kappa=0$, then $H_{2}=0$, and if $\kappa=-6 \lambda$ then since $H_{1}=-\frac{11}{3} \lambda$ is assumed to be constant, we get that $H_{2}$ is constant and then $e_{3}\left(H_{2}\right)=0$. Which is a contradiction, so we have $e_{3}\left(H_{2}\right)=0$.

Theorem 3.10. Let $x: M_{1}^{3} \rightarrow \mathbb{E}_{1}^{4}$ be an $L_{1}$-biharmonic timlike hypersurface with shape operator of type II in $\mathbb{E}_{1}^{4}$. If $M_{1}^{3}$ has constant mean curvature, then it is 1-minimal.

Proof. By assumption $H_{1}$ is assumed to be constant and then, by Proposition 3.9 it is proved that $H_{2}$ has to be constant. We claim that $H_{2}$ is null. Since the shape operator is of type II, there exist two possible cases as:

Case 1: $M_{1}^{3}$ has a principal curvature $\kappa$ of multiplicity 3;

Case 2: $M_{1}^{3}$ has two principal curvatures $\kappa$ and $\lambda$ of multiplicities 2 and 1 , respectively.

In Case 1, we have $H_{1}=\kappa, H_{2}=\kappa^{2}$ and $H_{3}=\kappa^{3}$. By [(2.9)(i)], we have $3 H_{1} H_{2}^{2}=H_{2} H_{3}$, which gives $\kappa^{5}=0$, and then $H_{2}=0$. 
In Case 2, we have $H_{1}=\frac{1}{3}(2 \kappa+\lambda), H_{2}=\frac{1}{3}\left(\kappa^{2}+2 \kappa \lambda\right)$ and $H_{3}=\kappa^{2} \lambda$. We assume that $H_{2} \neq 0$ and continue in two subcases as follow. Since $H_{2} \neq 0$, then $\kappa \neq 0$ and by using [(2.9)(i)] we obtain that $H_{3}$ is constant. Therefore, all of mean curvatures $H_{i}$ (for $i=1,2,3$ ) are constant, which means that $M_{1}^{3}$ is isoparametric. By Corollary 2.7 in [14], an isoparametric Lorentzian hypersurface of Case II in the Einstein space has at most one nonzero principal curvature, so we get $\lambda=0$. Then $H_{1}=\frac{2}{3} \kappa, H_{2}=\frac{1}{3} \kappa^{2}$ and $H_{3}=0$, hence, by [(2.9))(i)], we get $\kappa=0$, which contradicts with the assumption of this case. Therefore $\mathrm{H}_{2}=0$.

\subsection{Timelike hypersurfeces of types III}

Proposition 3.11. Let $x: M_{1}^{3} \rightarrow \mathbb{E}_{1}^{4}$ be an $L_{1}$-biharmonic connected orientable timelike hypersurface with shape operator of type III in $\mathbb{E}_{1}^{4}$. Then $M_{1}^{3}$ has constant 2 nd mean curvature.

Proof. Suppose that, $H_{2}$ be non-constant. Considering the open subset $\mathscr{U}=\left\{p \in M: \nabla H_{2}^{2}(p) \neq\right.$ $0\}$, we try to show $\mathscr{U}=\varnothing$. By the assumption, with respect to a suitable (local) orthonormal tangent frame $\left\{e_{1}, e_{2}, e_{3}\right\}$ on $M$, the shape operator $S$ has the matrix form $\tilde{B}_{3}$, such that $S e_{1}=\kappa e_{1}+\frac{\sqrt{2}}{2} e_{3}, S e_{2}=\kappa e_{2}-\frac{\sqrt{2}}{2} e_{3}, S e_{3}=-\frac{\sqrt{2}}{2} e_{1}-\frac{\sqrt{2}}{2} e_{2}+\kappa e_{3}$ and then, we have $P_{2} e_{1}=$ $\left(\kappa^{2}-\frac{1}{2}\right) e_{1}-\frac{1}{2} e_{2}-\frac{\sqrt{2}}{2} \kappa e_{3}, P_{2} e_{2}=\frac{1}{2} e_{1}+\left(\kappa^{2}+\frac{1}{2}\right) e_{2}+\frac{\sqrt{2}}{2} \kappa e_{3}$ and $P_{2} e_{3}=\frac{\sqrt{2}}{2} \kappa e_{1}+\frac{\sqrt{2}}{2} \kappa e_{2}+\kappa^{2} e_{3}$.

Using the polar decomposition $\nabla H_{2}=\sum_{i=1}^{3} \epsilon_{i} e_{i}\left(H_{2}\right) e_{i}$, from condition (2.9(ii)) we get

(i) $\epsilon_{1} e_{1}\left(H_{2}\right)\left[\left(\kappa^{2}-\frac{1}{2}\right)-\frac{9}{2} H_{2}\right]+\frac{1}{2} \epsilon_{2} e_{2}\left(H_{2}\right)+\frac{\sqrt{2}}{2} \epsilon_{3} e_{3}\left(H_{2}\right) \kappa=0$

(ii) $\frac{-1}{2} \epsilon_{1} e_{1}\left(H_{2}\right)+\epsilon_{2} e_{2}\left(H_{2}\right)\left[\left(\kappa^{2}+\frac{1}{2}\right)-\frac{9}{2} H_{2}\right]+\frac{\sqrt{2}}{2} \epsilon_{3} e_{3}\left(H_{2}\right) \kappa=0$

(iii) $\epsilon_{1} e_{1}\left(H_{2}\right) \frac{-\sqrt{2}}{2} \kappa+\epsilon_{2} e_{2}\left(H_{2}\right) \frac{\sqrt{2}}{2} \kappa+\epsilon_{3} e_{3}\left(H_{2}\right)\left(\kappa^{2}-\frac{9}{2} H_{2}\right)=0$.

Now, we prove some simple claims.

Claim: $e_{1}\left(H_{2}\right)=e_{2}\left(H_{2}\right)=e_{3}\left(H_{2}\right)=0$.

If $e_{1}\left(H_{2}\right) \neq 0$, then by dividing both sides of equalities [(3.44)(i, ii, iii)] by $\epsilon_{1} e_{1}\left(H_{2}\right)$, and using the identity $\mathrm{H}_{2}=\kappa^{2}$ in Case III, we get
(i) $-\frac{1}{2}-\frac{7}{2} \kappa^{2}+\frac{1}{2} u_{1}+\frac{\sqrt{2}}{2} u_{2} \kappa=0$
(ii) $\frac{-1}{2}+u_{1}\left(\frac{1}{2}-\frac{7}{2} \kappa^{2}\right)+\frac{\sqrt{2}}{2} u_{2} \kappa=0$
(i) $\left.\frac{-\sqrt{2}}{2} \kappa+\frac{\sqrt{2}}{2} u_{1} \kappa-\frac{7}{2} \kappa^{2}\right) u_{2}=0$,

where $u_{1}:=\frac{\epsilon_{2} e_{2}\left(H_{2}\right)}{\epsilon_{1} e_{1}\left(H_{2}\right)}$ and $u_{2}:=\frac{\epsilon_{3} e_{3}\left(H_{2}\right)}{\epsilon_{1} e_{1}\left(H_{2}\right)}$, which, by comparing (i) and (ii), gives $\kappa^{2}\left(u_{1}-1\right)=0$. If $\kappa=0$, then $H_{2}=0$. Assuming $\kappa \neq 0$, we get $u_{1}=1$, which, using [(3.47)(iii)], gives $u_{2}=0$. 
Substituting $u_{1}=1$ and $u_{2}=0$ in [(3.47)(i)], we obtain again $\kappa=0$, which is a contradiction. Hence $e_{1}\left(H_{2}\right) \equiv 0$.

Therefore, using the result $e_{1}\left(H_{2}\right) \equiv 0$, the system of equations (3.46) gives
(i) $\frac{1}{2} \epsilon_{2} e_{2}\left(H_{2}\right)+\frac{\sqrt{2}}{2} \epsilon_{3} e_{3}\left(H_{2}\right) \kappa=0$
(ii) $\epsilon_{2} e_{2}\left(H_{2}\right)\left(\frac{1}{2}-\frac{7}{2} \kappa^{2}\right)+\frac{\sqrt{2}}{2} \epsilon_{3} e_{3}\left(H_{2}\right) \kappa=0$
(iii) $\epsilon_{2} e_{2}\left(H_{2}\right) \frac{\sqrt{2}}{2} \kappa-\epsilon_{3} e_{3}\left(H_{2}\right) \frac{7}{2} \kappa^{2}=0$.

Comparing (i) and (ii), we get $\kappa e_{2}\left(H_{2}\right)=0$, which using i i $i$ gives $\kappa e_{3}\left(H_{2}\right)=0$, and then, using (i), gives $e_{2}\left(H_{2}\right)=0$. Then, the second claim (i.e. $e_{2}\left(H_{2}\right)=0$ ) is proved.

Now, using the results $e_{1}\left(H_{2}\right)=e_{2}\left(H_{2}\right)=0$, we get $\kappa e_{3}\left(H_{2}\right)=0$, which, using $H_{2}=\kappa^{2}$, implies $\kappa e_{3}\left(\kappa^{2}\right)=0$ and then $e_{3}\left(\kappa^{3}\right)=0$, and finally $e_{3}\left(H_{2}\right)=0$.

Theorem 3.12. Let $x: M_{1}^{3} \rightarrow \mathbb{E}_{1}^{4}$ be a $L_{1}$-biharmonic timlike hypersurface with shape operator of type III in $\mathbb{E}_{1}^{4}$. Then, it is 1-minimal. Furthermore, all of mean curvatures of $M_{1}^{3}$ are null.

Proof. By Proposition 3.11, the 2th mean curvature of $M_{1}^{3}$ is constant, which, by [(2.9)(i)], gives $L_{1} H_{2}=9 H_{1} H_{2}^{2}-3 H_{2} H_{3}=0$, and then $3 H_{1} H_{2}^{2}=H_{2} H_{3}$, which, using $H_{1}=\kappa, H_{2}=\kappa^{2}$ and $H_{3}=\kappa^{3}$, gives $\kappa^{5}=0$, and then $H_{1}=H_{2}=H_{3}=0$.

\subsection{Timelike hypersurfeces of types IV}

Proposition 3.13. Let $x: M_{1}^{3} \rightarrow \mathbb{E}_{1}^{4}$ be an $L_{1}$-biharmonic connected orientable timelike hypersurface with shape operator of type IV in $\mathbb{E}_{1}^{4}$. If $M_{1}^{3}$ has constant mean curvature and a constant real principal curvature, then its 2 nd and 3 rd mean curvatures are constant.

Proof. Suppose that, $H_{2}$ be non-constant. Considering the open subset $\mathscr{U}=\left\{p \in M: \nabla H_{2}^{2}(p) \neq\right.$ $0\}$, we try to show $\mathscr{U}=\varnothing$. By the assumption $M_{1}^{3}$ has three distinct principal curvature, then, with respect to a suitable (local) orthonormal tangent frame $\left\{e_{1}, e_{2}, e_{3}\right\}$ on $M$, the shape operator $S$ has the matrix form $B_{4}$, such that $S e_{1}=\kappa e_{1}-\lambda e_{2}, S e_{2}=\lambda e_{1}+\kappa e_{2}, S e_{3}=\eta e_{3}$ and then, we have $P_{2} e_{1}=\kappa \eta e_{1}+\lambda \eta e_{2}, P_{2} e_{2}=-\lambda \eta e_{1}+\kappa \eta e_{2}$ and $P_{2} e_{3}=\left(\kappa^{2}+\lambda^{2}\right) e_{3}$.

Using the polar decomposition $\nabla H_{2}=\sum_{i=1}^{3} \epsilon_{i} e_{i}\left(H_{2}\right) e_{i}$, from condition (2.9(ii)) we get

(i) $\epsilon_{1} e_{1}\left(H_{2}\right)\left(\kappa \eta-\frac{9}{2} H_{2}\right)=\epsilon_{2} e_{2}\left(H_{2}\right) \lambda \eta$,

(ii) $\epsilon_{2} e_{2}\left(H_{2}\right)\left(\kappa \eta-\frac{9}{2} H_{2}\right)=-\epsilon_{1} e_{1}\left(H_{2}\right) \lambda \eta$,

(iii) $\epsilon_{3} e_{3}\left(H_{2}\right)\left(\kappa^{2}+\lambda^{2}-\frac{9}{2} H_{2}\right)=0$. 
Now, we prove three simple claims.

Claim 1: $e_{1}\left(H_{2}\right)=e_{2}\left(H_{2}\right)=0$.

If $e_{1}\left(H_{2}\right) \neq 0$, then by dividing both sides of equalities [(3.49) (i, ii)] by $\epsilon_{1} e_{1}\left(H_{2}\right)$ we get

$$
\begin{aligned}
& \text { (i) } \kappa \eta-\frac{9}{2} H_{2}=\frac{\epsilon_{2} e_{2}\left(H_{2}\right)}{\epsilon_{1} e_{1}\left(H_{2}\right)} \lambda \eta, \\
& \text { (ii) } \frac{\epsilon_{2} e_{2}\left(H_{2}\right)}{\epsilon_{1} e_{1}\left(H_{2}\right)}\left(\kappa \eta-\frac{9}{2} H_{2}\right)=-\lambda \eta,
\end{aligned}
$$

which, by substituting (i) in (ii), gives $\lambda \eta\left(1+\left(\frac{\epsilon_{2} e_{2}\left(H_{2}\right)}{\epsilon_{1} e_{1}\left(H_{2}\right)}\right)^{2}\right)=0$, then $\lambda \eta=0$. Since by assumption $\lambda \neq 0$, we get $\eta=0$. So, by [(3.50)(i)], we have $H_{2}=0$.

Similarly, if $e_{2}\left(H_{2}\right) \neq 0$, then by dividing both sides of equalities [(3.49) (i , ii)] by $\epsilon_{2} e_{2}\left(H_{2}\right)$ we get

$$
\begin{aligned}
& \text { (i) } \frac{\epsilon_{1} e_{1}\left(H_{2}\right)}{\epsilon_{2} e_{2}\left(H_{2}\right)}\left(\kappa \eta-\frac{9}{2} H_{2}\right)=\lambda \eta, \\
& \text { (ii) } \kappa \eta-\frac{9}{2} H_{2}=-\frac{\epsilon_{1} e_{1}\left(H_{2}\right)}{\epsilon_{2} e_{2}\left(H_{2}\right)} \lambda \eta,
\end{aligned}
$$

which, by substituting (i) in (ii), gives $\lambda \eta\left(1+\left(\frac{\epsilon_{1} e_{1}\left(H_{2}\right)}{\epsilon_{2} e_{2}\left(H_{2}\right)}\right)^{2}\right)=0$, then $\lambda \eta=0$. Since by assumption $\lambda \neq 0$, we get $\eta=0$. So, by [(3.51)(ii)], we have $H_{2}=0$.

Claim 2: $e_{3}\left(H_{2}\right)=0$.

If $e_{3}\left(H_{2}\right) \neq 0$, then from equality [(3.49) (iii)] we have $\kappa^{2}+\lambda^{2}=\frac{9}{2} H_{2}$, which gives $\kappa^{2}+\lambda^{2}=-6 \kappa \eta$, where $\eta=3 H_{1}-2 \kappa$ and $\eta$ and $H_{1}$ are assumed to be constant on $\mathscr{U}$. So, $\kappa$ is also constant on $\mathscr{U}$, and then, we obtain $H_{2}=\frac{-4}{3} \kappa \eta=\frac{8}{3} \kappa^{2}-4 H_{1} \kappa$ and $H_{3}=-6 \kappa \eta^{2}=-6 \kappa\left(3 H_{1}-2 \kappa\right)^{2}$. are constant on $\mathscr{U}$.

Theorem 3.14. Let $x: M_{1}^{3} \rightarrow \mathbb{E}_{1}^{4}$ be an $L_{1}$-biharmonic connected orientable timelike hypersurface with shape operator of type IV in $\mathbb{E}_{1}^{4}$. If $M_{1}^{3}$ has constant mean curvature and a constant real principal curvature, then it is 1-minimal.

Proof. By Proposition 3.13, the 2th mean curvature of $M_{1}^{3}$ is constant, which gives $L_{1} H_{2}=0$. Then, by [(2.9)(i)], we have $9 H_{1} H_{2}^{2}-3 H_{2} H_{3}=0$, which gives $(7 \eta-4 \kappa) \kappa^{2} \eta^{2}=0$.

Now, if $7 \eta=4 \kappa$, then from $\kappa^{2}+\lambda^{2}=-6 \kappa \eta$ we get $\frac{31}{7} \kappa^{2}+\lambda^{2}=0$, and then $\kappa=\lambda=0$, which gives $H_{2}=H_{3}=0$. Also, if $\kappa^{2} \eta^{2}=0$, then we have $H_{2}=H_{3}=0$.

\section{Acknowledgements}

The author would like to gratefully thank the anonymous referees for their careful reading of the paper and the corrections. 


\section{References}

[1] K. Akutagawa and S. Maeta, Biharmonic properly immersed submanifolds in Euclidean spaces, Geom. Dedicata, 164 (2013), 351-355.

[2] L. J. Alias, Gürbüz, N., An extension of Takahashi theorem for the linearized operators of the higher order mean curvatures, Geom. Dedicata, 121 (2006), 113-127.

[3] A. Arvanitoyeorgos, F. Defever, G. Kaimakamis and B. J. Papantoniou, Biharmonic Lorentz hypersurfaces in $E_{1}^{4}$, Pacific J. Math., 229 (2007), 293-306.

[4] A. Arvanitoyeorgos, F. Defever and G. Kaimakamis, Hypersurfaces in $E_{s}^{4}$ with proper mean curvature vector, J. Math. Soc. Japan, 59 (2007), 797-809.

[5] B. Y. Chen, Total Mean Curvature and Submanifolds of Finite Type, Series in Pure Mathematics, 2. World Scientific Publishing Co, Singapore, 2014.

[6] B. Y. Chen, Some open problems and conjetures on submanifolds of finite type, Soochow J. Math., 17 (1991), 169-188.

[7] F. Defever, Hypersurfaces of $E^{4}$ satisfying $\Delta \vec{H}=\lambda \vec{H}$, Michigan. Math. J., 44 (1997), 355-363.

[8] F. Defever, Hypersurfaces of $E^{4}$ with harmonic mean curvature vector, Math. Nachr., 196 (1998), 61-69.

[9] I. Dimitrić, Submanifolds of $E^{n}$ with harmonic mean curvature vector, Bull. Inst. Math. Acad. Sin., 20 (1992), 53-65.

[10] J. Eells and J. C. Wood, Restrictions on harmonic maps of surfaces, Topology, 15 (1976), 263-266.

[11] T. Hasanis and T. Vlachos, Hypersurfaces in $E^{4}$ with harmonic mean curvature vector field, Math. Nachr., 172 (1995), 145-169.

[12] S. M. B. Kashani, On some $L_{1}$-finite type (hyper)surfaces in $\mathbb{R}^{n+1}$, Bull. Korean Math. Soc., 46 (2009), $35-43$.

[13] P. Lucas and H. F. Ramirez-Ospina, Hypersurfaces in the Lorentz-Minkowski space satisfying $L_{k} \psi=A \psi+b$, Geom. Dedicata, 153 (2011), 151-175.

[14] M. A. Magid, Lorentzian isoparametric hypersurfaces, Pacific J. of Math., 118 (1985), 165-197.

[15] F. Pashaie and A. Mohammadpouri, $L_{k}$-biharmonic spacelike hypersurfaces in Minkowski 4 -space $\mathbb{E}_{1}^{4}$, Sahand Comm. Math. Anal., 5 (2017), 21-30.

[16] B. O’Neill, Semi-Riemannian Geometry with Applicatins to Relativity, Acad. Press Inc., 1983.

[17] F. Pashaie and S. M. B. Kashani, Spacelike hypersurfaces in Riemannian or Lorentzian space forms satisfying $L_{k} x=A x+b$, Bull. Iran. Math. Soc., 39 (2013), 195-213.

[18] F. Pashaie and S. M. B. Kashani, Timelike hypersurfaces in the Lorentzian standard space forms satisfying $L_{k} x=A x+b$, Mediterr. J. Math., 11 (2014), 755-773.

[19] A. Z. Petrov, Einstein Spaces, Pergamon Press, Hungary, Oxford and New York, 1969.

[20] R. C. Reilly, Variational properties of functions of the mean curvatures for hypersurfaces in space forms, J. Differential Geom., 8 (1973), 465-477.

Department of Mathematics, Faculty of Basic Sciences, University of Maragheh, P.O.Box 55181-83111, Maragheh, Iran.

E-mail: f_pashaie@maragheh.ac.ir 